PROCEEDINGS OF THE

AMERICAN MATHEMATICAL SOCIETY

Volume 129, Number 10, Pages 2965-2972

S 0002-9939(01)06035-X

Article electronically published on March 15, 2001

\title{
FUGLEDE'S CONJECTURE FOR A UNION OF TWO INTERVALS
}

\author{
I. $\mathrm{AABA}$ \\ (Communicated by Christopher D. Sogge)
}

\begin{abstract}
We prove that a union of two intervals in $\mathbf{R}$ is a spectral set if and only if it tiles $\mathbf{R}$ by translations.
\end{abstract}

\section{The RESUlts}

A Borel set $\Omega \subset \mathbf{R}^{n}$ of positive measure is said to tile $\mathbf{R}^{n}$ by translations if there is a discrete set $T \subset \mathbf{R}^{n}$ such that, up to sets of measure 0 , the sets $\Omega+t, t \in T$, are disjoint and $\bigcup_{t \in T}(\Omega+t)=\mathbf{R}^{n}$. We may rescale $\Omega$ so that $|\Omega|=1$. We say that $\Lambda=\left\{\lambda_{k}: k \in \mathbf{Z}\right\} \subset \mathbf{R}^{n}$ is a spectrum for $\Omega$ if

$$
\left\{e^{2 \pi i \lambda_{k} \cdot x}\right\}_{k \in \mathbf{Z}} \text { is an orthonormal basis for } L^{2}(\Omega) .
$$

A spectral set is a domain $\Omega \subset \mathbf{R}^{n}$ such that (1.1) holds for some $\Lambda$.

Fuglede [2] conjectured that a domain $\Omega \subset \mathbf{R}^{n}$ is a spectral set if and only if it tiles $\mathbf{R}^{n}$ by translations, and proved this conjecture under the assumption that either $\Lambda$ or $T$ is a lattice. The conjecture is related to the question of the existence of commuting self-adjoint extensions of the operators $-i \frac{\partial}{\partial x_{j}}, j=1, \ldots, n[2],[7]$, [17]; other relations between the tiling and spectral properties of subsets of $\mathbf{R}^{n}$ have been conjectured and, in some cases, proved; see [6], [8, 9], [11, [12, 15].

Recently there has been significant progress on the special case of the conjecture when $\Omega$ is assumed to be convex [10, [3, [4, and in particular the 2-dimensional convex case appears to be nearly resolved [5]. The non-convex case is considerably more complicated, and is not understood even in dimension 1 . The strongest results yet in that direction seem to be those of Lagarias and Wang [14, [15], who proved that all tilings of $\mathbf{R}$ by a bounded region must be periodic, and that the corresponding translation sets are rational up to affine transformations. This in turn leads to a structure theorem for bounded tiles. It was also observed in [15] that the "tiling implies spectrum" part of Fuglede's conjecture for compact sets in $\mathbf{R}$ would follow from a conjecture of Tijdeman [20] concerning factorization of finite cyclic groups; however, Tijdeman's conjecture is now known to fail without additional assumptions (see [13] for a discussion). See also [16, [1] for partial results on the related problem of characterizing all tilings of $\mathbf{Z}$ by a finite set, and [15], [18 for a classification of domains in $\mathbf{R}^{n}$ which have $L+\mathbf{Z}^{n}$ as a spectrum for some finite

Received by the editors February 16, 2000.

2000 Mathematics Subject Classification. Primary 42A99. 
set $L$. Another recent result [19] is that sets which tile $[0, \infty)$ by translations are spectral sets.

The purpose of the present article is to address the following special case of Fuglede's conjecture in one dimension. Let $\Omega=I_{1} \cup I_{2}$, where $I_{1}, I_{2}$ are disjoint intervals of non-zero length. By scaling, translation, and symmetric reflection, we may assume that

$$
\Omega=(0, r) \cup(a, a+1-r), 0<r \leq \frac{1}{2}, a \geq r .
$$

Our first theorem characterizes all $\Omega$ 's of the form (1.2) which are spectral sets.

Theorem 1.1. Suppose that $\Lambda$ is a spectrum for $\Omega, 0 \in \Lambda$. Then at least one of the following holds:

(i) $a-r \in \mathbf{Z}$ and $\Lambda=\mathbf{Z}$;

(ii) $r=\frac{1}{2}, a=\frac{n}{2}$ for some $n \in \mathbf{Z}$, and $\Lambda=2 \mathbf{Z} \bigcup\left(\frac{p}{n}+2 \mathbf{Z}\right)$ for some odd integer $p$. Conversely, if $\Omega$ and $\Lambda$ satisfy (1.2) and if either (i) or (ii) holds, then $\Lambda$ is a spectrum for $\Omega$.

As a corollary, we prove that Fuglede's conjecture holds for a union of two intervals.

Theorem 1.2. Let $\Omega \subset \mathbf{R}$ be a union of two disjoint intervals, $|\Omega|=1$. Then $\Omega$ has a spectrum if and only if it tiles $\mathbf{R}$ by translations.

Theorem 1.2 follows easily from Theorem 1.1 We may assume that $\Omega$ is as in (1.2). Suppose that $\Lambda$ is a spectrum for $\Omega$; without loss of generality, we may assume that $0 \in \Lambda$. Then by Theorem 1.1 one of the conclusions (i), (ii) must hold, and in each of these cases $\Omega$ tiles $\mathbf{R}$ by translations. Conversely, if $\Omega$ tiles $\mathbf{R}$ by translations, by Proposition $2.1 \Omega$ must satisfy Theorem 1.1(i) or (ii), hence by Theorem 1.1 again it is a spectral set.

Theorem 1.1] will be proved as follows. Suppose that $\Lambda=\left\{\lambda_{k}: k \in \mathbf{Z}\right\}$ is a spectrum for $\Omega$; we may assume that $\lambda_{0}=0$. Let $\lambda_{k k^{\prime}}=\lambda_{k}-\lambda_{k^{\prime}}, \Lambda-\Lambda=\left\{\lambda_{k k^{\prime}}\right.$ : $\left.k, k^{\prime} \in \mathbf{Z}\right\}$, and

$$
Z_{\Omega}=\{0\} \cup\left\{\lambda \in \mathbf{R}: \hat{\chi}_{\Omega}(\lambda)=0\right\} .
$$

Then the functions $e^{2 \pi i \lambda_{k} x}$ are mutually orthogonal in $L^{2}(\Omega)$, hence $\Lambda \subset \Lambda-\Lambda \subset$ $Z_{\Omega}$. This will lead to a number of restrictions on the possible values of $\lambda_{k}$. Next, let

$$
\phi_{\lambda}(x)=\chi_{(0, r)} e^{2 \pi \lambda i x}
$$

where $\chi_{(0, r)}$ denotes the characteristic function of $(0, r)$. By Parseval's formula, the Fourier coefficients $c_{k}=\int_{0}^{r} e^{2 \pi i\left(\lambda-\lambda_{k}\right) x} d x$ of $\phi_{\lambda}$ satisfy

$$
\sum_{k \in \mathbf{Z}} c_{k}^{2}=\left\|\chi_{(0, r)} e^{2 \pi i \lambda x}\right\|_{L^{2}(\Omega)}^{2}=r .
$$

Given that the $\lambda_{k}$ 's are subject to the orthogonality restrictions mentioned above, we will find that there are not enough $\lambda_{k}$ 's for (1.5) to hold, unless the conditions of Theorem 1.1 are satisfied.

The author is grateful to Alex Iosevich for helpful conversations about spectral sets and Fuglede's conjecture, and to Jeffrey Lagarias and Yang Wang for bringing the references [13] and [19] to her attention. 


\section{TILING IMPLIES SPECTRUM}

Proposition 2.1. If $\Omega$ as in (1.2) tiles $\mathbf{R}$ by translations, it must satisfy (i) or (ii) of Theorem 1.1.

Proof. Suppose that $\mathbf{R}$ may be tiled by translates of $\Omega$. Assume first that $r=\frac{1}{2}$. Then any copy of $\Omega$ used in the tiling has a "gap" of length $a-r=a-\frac{1}{2}$, which must be covered by non-overlapping intervals of length $\frac{1}{2}$; hence $a \in \frac{1}{2} \mathbf{Z}$ as in Theorem 1.1(ii).

Assume now that $0<r<\frac{1}{2}$. Let $I_{1}=(0, r), I_{2}=(a, a+1-r)$. We will prove that translates of $I_{1}$ and $I_{2}$ must alternate in any tiling $\mathcal{T}$ of $\mathbf{R}$ by translates of $\Omega$; this implies immediately that $a-r \in \mathbf{Z}$ as in Theorem $1.1 \mathrm{i}$ ).

- If $\mathcal{T}$ contained two consecutive translates $(\tau, \tau+r)$ and $(\tau+r, \tau+2 r)$ of $I_{1}$, it would also contain the matching translates $(\tau+a, \tau+a+1-r)$ and $(\tau+a+r, \tau+a+1)$ of $I_{2}$, which is impossible since the latter two intervals overlap.

- Suppose now that $\mathcal{T}$ contains two consecutive translates $(\tau+a, \tau+a+1-r)$ and $(\tau+a+1-r, \tau+a+2-2 r)$ of $I_{2}$. Then $\mathcal{T}$ must also contain the matching translates $I_{1}^{\prime}=(\tau, \tau+r)$ and $I_{1}^{\prime \prime}=(\tau+1-r, \tau+2-2 r)$ of $I_{1}$. The gap between $I_{1}^{\prime}$ and $I_{1}^{\prime \prime}$ has length $1-2 r$, which is strictly less than $1-r=\left|I_{2}\right|$, so that $I_{1}^{\prime}$ must be followed by another translate of $I_{1}$. But this has just been shown to be impossible.

Next, we prove the second part of Theorem 1.1. This easy result appears to have been known to several authors; see, e.g., the examples in [2], [8], [15]. Since we will rely on it later on in the proof of the "hard" part of the theorem, we include the short proof.

Proposition 2.2. If $\Lambda$ and $\Omega$ are as in Theorem 1.1 $\mathrm{i}$ ) or (ii), then $\Lambda$ is a spectrum for $\Omega$.

Proof. If (i) holds, then $\Omega$ is a fundamental domain for $\mathbf{Z}$, and, consequently, $\Lambda=\mathbf{Z}$ is a spectrum [2]. Suppose now that (ii) holds. For any function $f$ on $\Omega$, we define functions $f_{+}, f_{-}$:

$$
f_{+}(x)=\frac{1}{2}\left(f(x)+f\left(x^{\prime}\right)\right), f_{-}(x)=\frac{1}{2}\left(f(x)-f\left(x^{\prime}\right)\right), x \in \Omega,
$$

where $x^{\prime}=x+a$ if $x \in\left(0, \frac{1}{2}\right)$, and $x^{\prime}=x-a$ if $x \in\left(a, a+\frac{1}{2}\right)$. Then

$$
f(x)=f_{+}(x)+f_{-}(x), f_{+}(x)=f_{+}\left(x^{\prime}\right), f_{-}(x)=-f_{-}\left(x^{\prime}\right) .
$$

It therefore suffices to prove that

$$
\begin{gathered}
g(x)=\sum_{k \in \mathbf{Z}} c_{k} e^{4 k \pi i x} \text { for any } g(x) \in L^{2}(\Omega) \text { such that } g(x)=g\left(x^{\prime}\right), \\
h(x)=\sum_{k \in \mathbf{Z}} c_{k}^{\prime} e^{\left(4 k+\frac{2 p}{n}\right) \pi i x} \text { for any } h(x) \in L^{2}(\Omega) \text { such that } h(x)=-h\left(x^{\prime}\right) .
\end{gathered}
$$

Since $e^{4 k \pi i x}, k \in \mathbf{Z}$, is a spectrum for $\left(0, \frac{1}{2}\right)$, we have

$$
g(x)=\sum_{k \in \mathbf{Z}} c_{k} e^{4 k \pi i x}, h(x)=e^{\frac{2 p}{n} \pi i x} \sum_{k \in \mathbf{Z}} c_{k}^{\prime} e^{4 k \pi i x}, x \in\left(0, \frac{1}{2}\right) .
$$


(2.1) follows immediately by periodicity. From the second equation above we find that (2.2) holds for all $x \in\left(0, \frac{1}{2}\right)$, and that for such $x$

$$
e^{\frac{2 p}{n} \pi i(x+a)} \sum_{k \in \mathbf{Z}} c_{k}^{\prime} e^{4 k \pi i(x+a)}=-e^{\frac{2 p}{n} \pi i x} \sum_{k \in \mathbf{Z}} c_{k}^{\prime} e^{4 k \pi i x}=-h(x)=h(x+a),
$$

where we used that $\frac{2 p}{n} a=p$ is odd. Hence, (2.2) also holds for $x \in\left(a, a+\frac{1}{2}\right)$. This ends the proof of Proposition 2.2 .

\section{Orthogonality}

We now begin the proof of the first part of Theorem 1.1 Throughout the rest of the paper, $\Omega$ is assumed to satisfy (1.2), $\Lambda=\left\{\lambda_{k}: k \in \mathbf{Z}\right\}$ is a spectrum for $\Omega$, $\lambda_{0}=0, \lambda_{k k^{\prime}}=\lambda_{k}-\lambda_{k^{\prime}}, \Lambda-\Lambda=\left\{\lambda_{k k^{\prime}}: k, k^{\prime} \in \mathbf{Z}\right\}$, and $Z_{\Omega}$ is defined by (1.3).

Lemma 3.1. $Z_{\Omega}=Z_{1} \cup Z_{2} \cup Z_{3}$, where

$$
\begin{aligned}
& Z_{1}=\left\{\lambda \in \mathbf{R}: \lambda a \in \mathbf{Z}+\frac{1}{2}, \lambda(2 r-1) \in \mathbf{Z}\right\}, \\
& Z_{2}=\{\lambda \in \mathbf{Z}: \lambda r \in \mathbf{Z}\} \\
& Z_{3}=\{\lambda \in \mathbf{Z}: \lambda(a-r) \in \mathbf{Z}\} .
\end{aligned}
$$

Proof. Suppose that $\lambda \neq 0, \lambda \in Z_{\Omega}$. Then

$$
\int_{\Omega} e^{2 \pi i \lambda x} d x=e^{2 \pi i \lambda r}-1+e^{2 \pi i \lambda(a+1-r)}-e^{2 \pi i \lambda a}=0 .
$$

All solutions to $z_{1}+z_{2}+z_{3}+1=0,\left|z_{i}\right|=1$, must be of the form $\left\{z_{1}, z_{2}, z_{3}\right\}=$ $\left\{-1, z_{*},-z_{*}\right\}$. Hence, $\lambda \in Z_{\Omega}$ if and only if one of the following holds:

- $e^{2 \pi i \lambda a}=-1$ and $e^{2 \pi i \lambda r}+e^{2 \pi i \lambda(a+1-r)}=0$, hence $\lambda \in Z_{1}$;

- $e^{2 \pi i \lambda r}=1$ and $e^{2 \pi i \lambda(1-r)}=1$, hence $\lambda \in Z_{2}$;

- $e^{2 \pi i \lambda(a+1-r)}=1$ and $e^{2 \pi i \lambda a}=e^{2 \pi i \lambda r}$, hence $\lambda \in Z_{3}$.

Observe that $Z_{2}, Z_{3}$ are additive subgroups of $\mathbf{Z}$.

Lemma 3.2. At least one of the following holds:

$$
\begin{aligned}
& \Lambda \subset Z_{1} \cup Z_{2}, \\
& \Lambda \subset Z_{1} \cup Z_{3} .
\end{aligned}
$$

Proof. By Lemma 3.1, $\Lambda \subset \Lambda-\Lambda \subset Z_{\Omega} \subset Z_{1} \cup Z_{2} \cup Z_{3}$. If $Z_{2} \subset Z_{3}$, (3.2) holds. Suppose therefore that there is a $\lambda_{i} \in Z_{2} \backslash Z_{3}$. It suffices to prove that for any $\lambda_{j} \in Z_{3}$ we must have $\lambda_{j} \in Z_{1}$ or $\lambda_{j} \in Z_{2}$.

Let $\lambda_{j} \in Z_{3}$, then $\lambda_{i j}=\lambda_{i}-\lambda_{j} \in Z_{\Omega}$ by orthogonality. By Lemma 3.1 $\lambda_{i j} \in Z_{1} \cup Z_{2} \cup Z_{3}$. If $\lambda_{i j} \in Z_{2}$, then $\lambda_{j} \in Z_{2}$ and we are done, and if $\lambda_{i j} \in Z_{3}$, then $\lambda_{i} \in Z_{3}$, which contradicts the above assumption on $\lambda_{i}$. Therefore assume that $\lambda_{i j} \in Z_{1}$. Then

$$
\lambda_{i j} \in \mathbf{Z}, \lambda_{i j} a \in \mathbf{Z}+\frac{1}{2}, \lambda_{i j}(2 r-1) \in \mathbf{Z},
$$

hence

$$
2 \lambda_{j} r=2 \lambda_{i} r-\lambda_{i j}(2 r-1)-\lambda_{i j} \in \mathbf{Z} .
$$

If $\lambda_{j} r \in \mathbf{Z}$, then $\lambda_{j} \in Z_{2}$; if $\lambda_{j} r \in \mathbf{Z}+\frac{1}{2}$, then $\lambda_{j} a \in \mathbf{Z}+\frac{1}{2}$ by the definition of $Z_{3}$ and $\lambda_{j}(2 r-1) \in \mathbf{Z}$, so that $\lambda_{j} \in Z_{1}$. 
Lemma 3.3. (i) $\Lambda \subset Z_{2}$ is not possible;

(ii) $\Lambda \subset Z_{3}$ is possible only if $a-r \in \mathbf{Z}$ and $\Lambda=Z_{3}=\mathbf{Z}$.

Proof. Suppose that $\Lambda \subset Z_{i}$ for $i=2$ or 3 . Since $Z_{i}$ is an additive subgroup of $\mathbf{Z}$, we must have $Z_{i}=p \mathbf{Z}$ for some integer $p>0$. Furthermore, if there was a $\lambda \in p \mathbf{Z} \backslash \Lambda$, we would have $\lambda_{k}-\lambda \in p \mathbf{Z}$ and $e^{2 \pi i \lambda x}$ would be orthogonal to $e^{2 \pi i \lambda_{k} x}$ for all $\lambda_{k} \in \Lambda$, which would contradict (1.1). Hence, $\Lambda=Z_{i}=p \mathbf{Z}$. We also observe that, if $p$ were $\geq 2$, any function of the form $f(x)=\sum_{k \in \mathbf{Z}} c_{k} e^{2 \pi i \lambda_{k} x}$ would be periodic with period $\frac{1}{p} \leq \frac{1}{2}$, which again would contradict (1.1). Thus $\Lambda=Z_{i}=\mathbf{Z}$.

If $i=2$, this is not possible, since $n r$ cannot be an integer for all $n \in \mathbf{Z}$ if $r \leq \frac{1}{2}$. If $i=3$, we obtain that $n(a-r) \in \mathbf{Z}$ for all $n \in \mathbf{Z}$; letting $n=1$, we find that $a-r \in \mathbf{Z}$.

If $\Omega, \Lambda$ are as in Lemma 3.3(ii), then Theorem 1.1(i) is satisfied and we are done. Thus we may assume throughout the sequel that

$$
\Lambda \not \subset Z_{2}, \Lambda \not \subset Z_{3} \text {. }
$$

Lemma 3.4. If (3.3) holds, then $\Lambda \subset Z_{1} \cup\left(Z_{2} \cap Z_{3}\right)$.

Proof. By Lemma 3.2 it suffices to prove that

$$
\begin{aligned}
& \text { if } \Lambda \cap\left(Z_{1} \backslash Z_{2}\right) \neq \emptyset \text {, then } \Lambda \cap Z_{2} \subset \Lambda \cap Z_{3} \text {; } \\
& \text { if } \Lambda \cap\left(Z_{1} \backslash Z_{3}\right) \neq \emptyset \text {, then } \Lambda \cap Z_{3} \subset \Lambda \cap Z_{2} ;
\end{aligned}
$$

We will only prove (3.4), since the proof of (3.5) is almost identical. Suppose that $\lambda_{i} \in Z_{1} \backslash Z_{2}$, and let $\lambda_{j} \in Z_{2}$. By Lemma 3.1, $\lambda_{i j}$ belongs to at least one of $Z_{1}, Z_{2}$, $Z_{3}$. We may not have $\lambda_{i j} \in Z_{2}$, since then $\lambda_{i}$ would also be in $Z_{2}$. Thus we only need consider the following two cases.

- Let $\lambda_{i j} \in Z_{1}$. Then $\lambda_{i} a, \lambda_{i j} a \in \mathbf{Z}+\frac{1}{2}$, hence $\lambda_{j} a \in \mathbf{Z}$ and $\lambda_{j} \in Z_{2} \cap Z_{3}$.

- Assume now that $\lambda_{i j} \in Z_{3}$. Then $\lambda_{i} \in \mathbf{Z}$, hence $2 \lambda_{i} r \in \mathbf{Z}$. Moreover, $\lambda_{i} r \in \mathbf{Z}$ would imply $\lambda_{i} \in Z_{2}$, hence $\lambda_{i} r \in \mathbf{Z}+\frac{1}{2}$. It follows that $\lambda_{i}(a-r) \in \mathbf{Z}$; since also $\lambda_{i j}(a-r) \in \mathbf{Z}$, we obtain that $\lambda_{j}(a-r) \in \mathbf{Z}$ and $\lambda_{j} \in Z_{2} \cap Z_{3}$.

Lemma 3.5. Assume (3.3). Then:

(i) $\Lambda-\Lambda \subset Z_{1} \cup\left(Z_{2} \cap Z_{3}\right)$;

(ii) $\Lambda \cap Z_{1} \subset \lambda_{*}+r^{-1} \mathbf{Z}$ for some $\lambda_{*} \in \mathbf{R}$.

Proof. For $k \in \mathbf{Z}$, let $\Lambda_{k}=\Lambda-\lambda_{k}=\left\{\lambda_{j k}: j \in \mathbf{Z}\right\}$. Then $\Lambda_{k}$ is also a spectrum for $\Omega, 0 \in \Lambda_{k}$, hence all of the results obtained so far apply with $\Lambda$ replaced by $\Lambda_{k}$. Thus (i) follows from Lemmas 3.3 and 3.4

To prove (ii), it suffices to verify that $\lambda_{i j} r \in \mathbf{Z}$ whenever $\lambda_{i}, \lambda_{j} \in Z_{1}$. Indeed, if $\lambda_{i}, \lambda_{j} \in Z_{1}$, then $\lambda_{i j} a \in \mathbf{Z}$, hence $\lambda_{i j} \notin Z_{1}$ and, by (i), $\lambda_{i j} \in Z_{2} \cap Z_{3}$. But this implies that $\lambda_{i j} r \in \mathbf{Z}$.

\section{Completeness}

Fix $j, n \in \mathbf{Z}$, and consider the function $\phi_{\lambda}$ defined by (1.4) with $\lambda=\lambda_{j}-n r^{-1}$. The Fourier coefficients of $\phi_{\lambda}$ are

$$
c_{k}=\int_{0}^{r} e^{2 \pi i\left(\lambda-\lambda_{k}\right) x} d x=\int_{0}^{r} e^{2 \pi i\left(\lambda_{j k}-n r^{-1}\right) x} d x
$$


hence $c_{k}=r$ if $\lambda_{j k}=n r^{-1}$, and

$$
c_{k}=\frac{1}{2 \pi i\left(\lambda_{j k}-n r^{-1}\right)}\left(e^{2 \pi i\left(\lambda_{j k} r-n\right)}-1\right), \lambda_{j k} \neq n r^{-1} .
$$

Define $\alpha_{j k}=\lambda_{j k} r$. Plugging (4.1) into (1.5), we obtain that for all $j \in \mathbf{Z}$,

$$
\frac{1}{r}=1+\sum_{k: \alpha_{j k} \notin \mathbf{Z}} \frac{1}{4 \pi^{2} \alpha_{j k}^{2}}\left|e^{2 \pi i \alpha_{j k}}-1\right|^{2},
$$

and for all $n, j \in \mathbf{Z}$,

$$
\frac{1}{r}=\delta_{n, j}+\sum_{k: \alpha_{j k} \notin \mathbf{Z}} \frac{1}{4 \pi^{2}\left(\alpha_{j k}-n\right)^{2}}\left|e^{2 \pi i\left(\alpha_{j k}-n\right)}-1\right|^{2},
$$

where $\delta_{n, j}=1$ if there is a $k \in \mathbf{Z}$ such that $\alpha_{j k}=n$, and $\delta_{n, j}=0$ otherwise.

We define the equivalence relation between the indices $k, k^{\prime}$ :

$$
k \sim k^{\prime} \Leftrightarrow \alpha_{k k^{\prime}} \in \mathbf{Z},
$$

and denote by $A_{1}, A_{2}, \ldots, A_{m}, \cdots \subset \mathbf{Z}$ the (non-empty and disjoint) equivalence classes with respect to this relation. Hence $k, k^{\prime}$ belong to the same $A_{m}$ if and only if $\alpha_{k k^{\prime}} \in \mathbf{Z}$; in particular,

$$
\mathcal{A}_{m}:=\left\{\alpha_{k}: k \in A_{m}\right\} \subset \beta_{m}+\mathbf{Z}
$$

for some $\beta_{m} \in[0,1)$.

Lemma 4.1. Let $M$ denote the number of distinct and non-empty $A_{m}$ 's. Then

$$
M \geq r^{-1} .
$$

Moreover, if one of the $\mathcal{A}_{m}$ 's skips a number (i.e. $\mathcal{A}_{m} \neq \beta_{m}+\mathbf{Z}$ ), then $M \geq r^{-1}+1$.

Proof. For each $m, m^{\prime}$, let $\beta_{m m^{\prime}}=\beta_{m}-\beta_{m^{\prime}}$; note that $\beta_{m m^{\prime}} \neq 0$ if $m \neq m^{\prime}$. Fix $m^{\prime}$ and $j \in A_{m^{\prime}}$, then (4.2) may be rewritten as

$$
\frac{1}{r}=1+\sum_{m \neq m^{\prime}} S_{m m^{\prime}}
$$

where

$$
S_{m m^{\prime}}=\sum_{k \in A_{m}} \frac{1}{4 \pi^{2} \alpha_{j k}^{2}}\left|e^{2 \pi i \beta_{m m^{\prime}}}-1\right|^{2}
$$

Clearly,

$$
S_{m m^{\prime}} \leq \tilde{S}\left(\beta_{m m^{\prime}}\right)
$$

where

$$
\tilde{S}(\beta)=\sum_{k \in \mathbf{Z}} \frac{1}{4 \pi^{2}(\beta+k)^{2}}\left|e^{2 \pi i \beta}-1\right|^{2} .
$$

Hence (4.4) follows from (4.5) and Lemma 4.2 below.

Suppose now that $\mathcal{A}_{m^{\prime}}$ skips a number. Then we may find $j \in A_{m^{\prime}}$ and $n \in \mathbf{Z}$ such that $\delta_{n, j}=0$, and (4.4) may be improved to $M \geq 1+r^{-1}$ by using (4.3) instead of (4.2).

Lemma 4.2. Let $\tilde{S}(\beta)$ be as in (4.7), then $\tilde{S}(\beta)=1$ for all $0<\beta<1$. 
Proof. By Proposition 2.2 $\Lambda=2 \mathbf{Z} \cup\left(\frac{p}{n}+2 \mathbf{Z}\right)$, where $n \in \mathbf{Z}$ and $p$ is an odd integer, is a spectrum for $\Omega=\left(0, \frac{1}{2}\right) \cup\left(\frac{n}{2}, \frac{n+1}{2}\right)$. Plugging this back into (4.2) we obtain that:

$$
1=\sum_{k \in \mathbf{Z}} \frac{1}{4 \pi^{2}(\beta+k)^{2}}\left|e^{2 \pi i \beta}-1\right|^{2}
$$

for $\beta=\frac{p}{2 n}$. However, the set of $\beta$ of this form is dense in $\mathbf{R}$, hence by continuity the lemma holds for all $\beta \in(0,1)$.

\section{Conclusion}

Proof of Theorem 1.1. If $\Lambda$ is as in Lemma 3.3(ii), then Theorem 1.1(i) is satisfied. We may therefore assume that (3.3) holds. From Lemma 3.5 we have:

$$
\Lambda-\Lambda \subset Z_{1} \cup\left(Z_{2} \cap Z_{3}\right), Z_{2} \cap Z_{3} \subset r^{-1} \mathbf{Z}, Z_{1} \subset\left(\lambda_{*}+r^{-1} \mathbf{Z}\right),
$$

for some $\lambda_{*} \in \mathbf{R}$, hence $M \leq 2$ (using the notation in Section 4 ). However, by Lemma $4.1 M \geq r^{-1} \geq 2$, and this may be improved to $M \geq 3$ if one of the $\mathcal{A}_{m}$ 's skips a number. Therefore we must have $r=\frac{1}{2}$, and

$$
\Lambda-\Lambda=2 \mathbf{Z} \cup\left(\lambda_{*}+2 \mathbf{Z}\right), Z_{2} \cap Z_{3}=2 \mathbf{Z}, Z_{1}=\lambda_{*}+2 \mathbf{Z} .
$$

Pick $\lambda_{i j}, \lambda_{k l} \in Z_{1}$ such that $\lambda_{i j}-\lambda_{k l}=2$. From the definition of $Z_{1}$ we have $\lambda_{i j} a, \lambda_{k l} a \in \mathbf{Z}+\frac{1}{2}$, hence

$$
2 a=\frac{a}{r}=\lambda_{i j} a-\lambda_{k l} a \in \mathbf{Z},
$$

so that $a=\frac{n}{2}$ for some $n \in \mathbf{Z}$. Finally, we have $\lambda_{*} a=\frac{1}{2} n \lambda_{*} \in \mathbf{Z}+\frac{1}{2}$, hence $\lambda_{*} n=p$ for some odd integer $p$. Thus $\Omega$ and $\Lambda$ satisfy (ii) of Theorem 1.1.

\section{REFERENCES}

[1] E. M. Coven, A. Meyerowitz: Tiling the integers with translates of one finite set, J. Algebra 212 (1999), 161-174. MR 99k:11032

[2] B. Fuglede: Commuting self-adjoint partial differential operators and a group theoretic problem, J. Funct. Anal. 16 (1974), 101-121. MR 57:10500

[3] A. Iosevich, N. H. Katz, S. Pedersen: Fourier bases and a distance problem of Erdös, Math. Res. Lett. 6 (1999), 251-255. MR 2000j:42013

[4] A. Iosevich, N. H. Katz, T. Tao: Convex bodies with a point of curvature do not have Fourier bases, Amer. J. Math, to appear.

[5] A. Iosevich, N. H. Katz, T. Tao: preprint in preparation.

[6] A. Iosevich, S. Pedersen: Spectral and tiling properties of the unit cube, Internat. Math. Res. Notices 16 (1998), 819-828. MR 2000d:52015

[7] P. Jorgensen: Spectral theory of finite volume domains in $\mathbf{R}^{n}$, Adv. Math. 44 (1982), 105120. MR 84k:47024

[8] P. Jorgensen, S. Pedersen: Spectral theory for Borel sets in $\mathbf{R}^{n}$ of finite measure, J. Funct. Anal. 107 (1992), 72-104. MR 93k:47005

[9] P. Jorgensen, S. Pedersen: Spectral pairs in Cartesian coordinates, J. Fourier Anal. Appl. 5 (1999), 285-302. CMP 99:15

[10] M. Kolountzakis: Non-symmetric convex domains have no basis of exponentials, Illinois J. Math. 44 (2000), 542-550. CMP 2000:16

[11] M. Kolountzakis: Packing, tiling, orthogonality, and completeness, Bull. London Math. Soc. 32 (2000), 589-599. CMP 2000:15

[12] J. C. Lagarias, J. A. Reed, Y. Wang: Orthonormal bases of exponentials for the $n$-cube, Duke Math. J. 103 (2000), 25-37. CMP 2000:12

[13] J. C. Lagarias, S. Szabó: Universal spectra and Tijdeman's conjecture on factorization of cyclic groups, J. Fourier Anal. Appl., to appear. 
[14] J. C. Lagarias, Y. Wang: Tiling the line with translates of one tile, Invent. Math. 124 (1996), 341-365. MR 96i:05040

[15] J. C. Lagarias, Y. Wang: Spectral sets and factorizations of finite abelian groups, J. Funct. Anal. 145 (1997), 73-98. MR 98b:47011b

[16] D. J. Newman: Tesselation of integers, J. Number Theory 9 (1977), 107-111. MR 55:2731

[17] S. Pedersen: Spectral theory of commuting self-adjoint partial differential operators, J. Funct. Anal. 73 (1987), 122-134. MR 89m:35163

[18] S. Pedersen: Spectral sets whose spectrum is a lattice with a base, J. Funct. Anal. 141 (1996), 496-509. MR 98b:47011a

[19] S. Pedersen, Y. Wang: Universal spectra, universal tiling sets and the spectral set conjecture, Scand. J. Math., to appear.

[20] R. Tijdeman: Decomposition of the integers as a direct sum of two subsets, London Math. Soc. Lecture Note Ser., vol.215, Cambridge Univ. Press, 1995, pp. 261-276. MR 96j:11025

Department of Mathematics, Princeton University, Princeton, New Jersey 08544

Current address: Department of Mathematics, University of British Columbia, Vancouver, Canada V6T $1 \mathrm{Z2}$

E-mail address: ilaba@math.ubc.ca 